\title{
MUC1 and maltose-binding protein recombinant fusion protein combined with Bacillus Calmette-Guerin induces MUC1-specific and nonspecific anti-tumor immunity in mice
}

\author{
FANG FANG ${ }^{1,2}$, JICHUN MA ${ }^{1,3}$, WEIHUA NI ${ }^{1}$, FENGLI WANG ${ }^{1}$, XIAXIA SUN ${ }^{1}$, YINGYING LI ${ }^{1}$, QIONGSHU LI ${ }^{1}$, \\ FEI XIE ${ }^{1}$, JUAN WANG ${ }^{1}$, RUIPING ZHAI ${ }^{1}$, ZHONGHUI LIU ${ }^{1}$, SUNJUN GAO ${ }^{1,4}$ and GUIXIANG TAI ${ }^{1}$ \\ ${ }^{1}$ Department of Immunology, Norman Bethune College of Medicine, Jilin University, Changchun, Jilin 130021; \\ ${ }^{2}$ Department of Immunology, Jilin Medical College, Jilin City, Jilin 132013; ${ }^{3}$ Affiliated People's Hospital, \\ Jiangsu University, Zhenjiang, Jiangsu 212000; ${ }^{4}$ Department of Hematology and Oncology, \\ The First Bethune Hospital of Jilin University, Changchun, Jilin 130021, P.R. China
}

Received September 15, 2013; Accepted March 17, 2014

DOI: $10.3892 / \mathrm{mmr} .2014 .2306$

\begin{abstract}
Human mucin 1 (MUC1) is a target for immunotherapy. The majorproblemassociated with MUC1-based cancer vaccines is the weakness of the immunogenicity of MUC1. The present study aimed to develop an efficient cancer vaccine through generating a recombinant fusion protein consisting of MUC1 and maltose-binding protein (MBP) by inserting seven tandem repeats encoding the human MUC1 gene into the pMAL-c2 expression vector. Bacillus Calmette-Guerin (BCG) was used as an adjuvant. MUC1 was found to predominantly induce T helper type 2 (Th2) cell responses. MUC1/BCG and MUC1-MBP were found to generate T helper (Th) type 1 and 2 responses, while MUC1-MBP/BCG induced a Th1 immune profile and stimulated MUC1-specific cytotoxic T lymphocyte killing activity. MUC1-MBP, as well as MBP and BCG alone were found to induce natural killer (NK) cell activity, with MUC1-MBP/BCG observed to synergistically induce NK cell activity. Furthermore, MUC1-MBP/BCG significantly inhibited $\mathrm{MUC1}^{+} \mathrm{B} 16$ cell growth in mice. These findings show that MBP augments the immunogenicity of MUC1 and that BCG enhances the efficacy of the MUC1-MBP vaccine. Thus, MUC1-MBP/BCG may have potential as a cancer vaccine for clinical application.
\end{abstract}

Correspondence to: Professor Guixiang Tai, Department of Immunology, Norman Bethune College of Medicine, Jilin University, 126 Xinmin Street, Changchun, Jilin 130021, P.R. China

E-mail: taiguixiang@163.com

Professor Sunjun Gao, Department of Hematology and Oncology, The First Bethune Hospital of Jilin University, 71 Xinmin Street, Changchun, Jilin 130021, P.R. China

E-mail: drsujung@gmail.yahoo.com.cn

Key words: Bacillus Calmette-Guerin, mucin 1, maltose-binding protein, mucin 1-maltose-binding protein, cancer vaccine, $\mathrm{T}$ cell

\section{Introduction}

Mucin 1 (MUC1) is a transmembrane glycoprotein which belongs to the human mucin family and consists of a serine- and threonine-rich protein core with highly branched carbohydrate side chains. The protein core of MUC1 is composed of extracellular, transmembrane and cytoplasmic domains (1). The extracellular domain contains a region of 20-125 tandem repeat sequences known as the variable number tandem repeat (VNTR) domain. The MUC1 tandem repeat sequence consists of the following 20 amino acids: SAPDTRPAPGSTAPPAHGVT (2). MUC1 is primarily expressed on epithelial cells and is aberrantly overexpressed in various epithelial-derived tumor cells, including breast, lung, ovarian, prostate and pancreatic tumors, as well as hematological malignancies (3-8). In normal cells, MUC1 is heavily glycosylated and is expressed exclusively at the apical surface of ductal cells (9). In tumor cells, the expression of underglycosylated MUC1 is greatly upregulated and it is distributed across the entire cell surface (10). Underglycosylated MUC1 in malignant cells unmasks novel peptide and carbohydrate epitopes. In addition, the PDTRP epitope of the MUC1 protein core has been shown to induce the production of specific MUC1 antibodies, as well as human leukocyte antigen-unrestricted cytotoxic $\mathrm{T}$ lymphocyte (CTL) activity against MUC1 (11). Thus, MUC1 is considered to be a target for tumor immunotherapy $(12,13)$. Numerous MUC1-based cancer vaccines have been developed in order to prevent adenocarcinoma growth through immune response induction. These vaccination approaches have included peptide and recombinant protein vaccines (14-16), as well as carbohydrate (17), DNA (18) and dendritic cell vaccines (19), with several of these vaccines entering clinical trials (20-22). However, some of these vaccines have been observed to elicit weak responses in humans, despite their antitumor responses in animal models. In order to augment the antitumor effect of these vaccines, numerous strategies have been developed to enhance their immunogenicity, including the use of various adjuvants, carrier proteins and viral vectors $(15,22,23)$. 
The Escherichia coli maltose-binding protein (MBP) is an $\sim 42 \mathrm{kDa}$, high affinity MBP protein responsible for binding and transporting maltose from the periplasmic space in gram-negative bacteria (24). Proteins of interest are frequently fused with MBP in order to improve their yield and facilitate purification (25). MBP has been utilized as a chaperone in various experimental vaccines, with recombinant protein-MBP found to enhance immunogenicity $(26,27)$. Previous studies by our group demonstrated that MBP promotes lymphocyte proliferation, directly activates T helper type 1 (Th1) and natural killer (NK) cells and enhances Bacillus Calmette-Guerin (BCG)-induced Th1 cell activation $(28,29)$. Therefore, it is hypothesized that an MBP-fused MUC1 protein may enhance the immunogenicity of MUC1. In order to investigate this hypothesis, in the present study, seven VNTRs encoding the human MUC1 core peptide (140 amino acids) were cloned into the pMAL-c2 expression vector to produce a recombinant MUC1-MBP fusion protein. The immunogenicity of the MUC1-MBP fusion protein was investigated by immunizing C57BL/6 mice with MUC1-MBP and assessing the antitumor activities using $\mathrm{MUC1}^{+}$and $\mathrm{MUC1}^{-}$tumor cell challenge models.

\section{Materials and methods}

Cell lines. The B16 mouse melanoma and YAC-1 NK-sensitive lymphoma cell lines (American Type Culture Collection, Rockville, MD, USA) were maintained in Iscove's Modified Dulbecco's Media (IMDM) containing 10\% fetal calf serum (Gibco-BRL, Carlsbad, CA, USA), $100 \mathrm{U} / \mathrm{ml}$ penicillin and $100 \mu \mathrm{g} / \mathrm{ml}$ streptomycin in a humidified atmosphere of $5 \% \mathrm{CO}_{2}$ at $37^{\circ} \mathrm{C}$. A stable human MUC1-expressing B16 (B16-MUC1) cell line was established. In brief, B16 cells were transfected with pcDNA3-MUC1 plasmids containing the full-length human MUC1 sequence consisting of 22 VNTRs. MUC1-positive clones were then selected using $1,000 \mu \mathrm{g} / \mathrm{ml} \mathrm{G} 418$ (Sigma-Aldrich, St. Louis, MO, USA) and MUC1 expression was assessed using flow cytometric analysis with anti-MUC1 monoclonal antibodies (clone, HMPV; BD Biosciences, San Jose, CA, USA). A B16-neo cell line was used as a negative control through transfecting B16 cells with an empty pcDNA3 plasmid.

Preparation of proteins and peptides. The cDNA fragment of the human MUC1 core peptide encoding seven VNTRs was cloned into pMAL-c2 plasmids (New England Biolabs, Beverly, MA, USA) to generate a MUC1-MBP fusion protein expression vector. Recombinant pMAL-MUC1 plasmids and empty pMAL-c2 plasmids were transformed into Escherichia coli DH5 $\alpha$. Expression of the MUC1-MBP fusion protein and the MBP protein was induced in Escherichia coli DH5 $\alpha$ cells using $0.1 \mathrm{mM}$ isopropyl- $\beta$-D-thiogalactopyranoside (IPTG; Sigma-Aldrich). MUC1-MBP and MBP were purified using amylose resin columns (New England Biolabs) as described previously (30). The MUC1 peptide was purified by cleaving the MUC1-MBP fusion protein using the Factor Xa protease (New England Biolabs) at $20^{\circ} \mathrm{C}$ for $48 \mathrm{~h}$. The purified MUC1 peptide, MUC1-MBP fusion protein and MBP protein were analyzed using $12 \%$ SDS-PAGE with Coomassie Brilliant Blue staining and detected using western blot analysis with
anti-MUC1 (GP1.4) and -MBP (dilution, 1:1,000) monoclonal antibodies (Neomarkers Inc., Freemont, CA, USA).

A synthetic MUC1 peptide, which was used in certain experiments and which corresponds to the SAPDTRPAPGSTAPPAHGVT tandem repeat, was synthesized by GL Biochem Ltd. (Shanghai, China) with $98 \%$ purity and termed the MUC1 synthetic peptide.

Mice and immunization. Female C57BL/6 mice, between six and eight weeks old, were purchased from the Norman Bethune Medical School of Jilin University (Changchun, China) and maintained under specific pathogen-free conditions. The experimental manipulation of mice was conducted in accordance with the National Institute of Health Guide for the Care and Use of Laboratory Animals and the approval of the Scientific Investigation Board of Science and Technology of Jilin Province (Changchun, China).

Mice were randomly divided into seven groups of five animals and were treated with the following agents: Phosphate-buffered saline (PBS) as a negative control, BCG (Shanghai Institute of Biological Products Co., Ltd., Shanghai, China) as an adjuvant control, MBP, MUC1, MUC1 with BCG (MUC1/BCG), MUC1-MBP and MUC1-MBP with BCG (MUC1-MBP/BCG). Mice were subcutaneously immunized with PBS, BCG (150 mg/kg), MBP (1.7 mg/kg), MUC1 (0.77 mg/kg), MUC1 (0.77 mg/kg)/BCG (150 mg/kg), MUC1-MBP (2.5 mg/kg) or MUC1-MBP (2.5 mg/kg)/BCG $(150 \mathrm{mg} / \mathrm{kg})$, three times at one-week intervals.

ELISA for MUC1-specific immunoglobulin G (IgG) subclasses. Five days after the final immunization, mouse serum was isolated and MUC1-specific antibodies were detected using ELISA. Briefly, 96-well plates were coated with $1 \mu \mathrm{g} /$ well MUC1 peptide and incubated overnight at $4^{\circ} \mathrm{C}$. Subsequent to blocking with PBS containing $2 \%$ bovine serum albumin, serum samples diluted 1:500 for total $\mathrm{IgG}$, IgG1 and IgG2c were added and incubated for $1.5 \mathrm{~h}$ at $37^{\circ} \mathrm{C}$. Following three washes with PBS containing $0.1 \%$ Tween ${ }^{\circledR} 20$, plates were incubated with horseradish peroxidase-labeled goat anti-mouse IgG, IgG1 and IgG2c for $1 \mathrm{~h}$ at $37^{\circ} \mathrm{C}$. Plates were then washed three times with PBS containing $0.1 \%$ Tween 20 and incubated with the substrate $o$-phenylenediamine for $10 \mathrm{~min}$. The reaction was terminated by adding $0.2 \mathrm{mM} \mathrm{H}_{2} \mathrm{SO}_{4}$. Absorbance (A) was measured at $490 \mathrm{~nm}$ using an ELISA reader (model 550; Bio-Rad Laboratories Inc., Hercules, CA, USA). Results were calculated as the A value from experimental groups minus the A value from the PBS negative control group.

Interferon (IFN)- $\gamma$ ELISA. Splenic mononuclear cells were cultured at a density of $5 \times 10^{5}$ cells/well in IMDM containing $100 \mathrm{U} / \mathrm{ml}$ interleukin (IL)-2 with or without $20 \mu \mathrm{g} / \mathrm{ml} \mathrm{MUC1}$ synthetic peptide at $37^{\circ} \mathrm{C}$ in $5 \% \mathrm{CO}_{2}$ for five days. The culture supernatants were then collected and IFN- $\gamma$ production was assessed using an ELISA kit (eBioscience, Inc., San Diego, CA, USA) according to the manufacturer's instructions. Cytokine levels were calculated as the cytokine levels detected upon stimulation with the MUC1 synthetic peptide minus the cytokine levels detected upon stimulation with the free MUC1 synthetic peptide. 
A

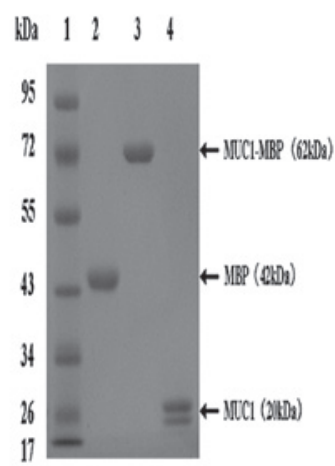

B

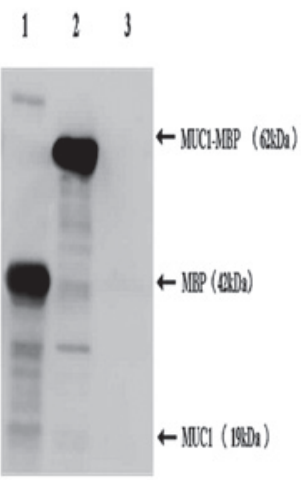

C

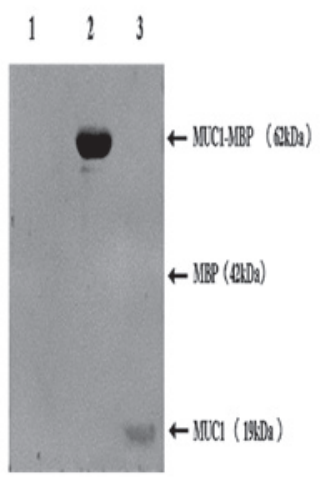

Figure 1. SDS-PAGE and western blot analysis of purified MUC1-MBP, MBP and MUC1 proteins. (A) Purified MUC1-MBP, MBP and MUC1 were separated using 12\% SDS-PAGE and stained with Coomassie Brilliant Blue. Lane 1, protein molecular weight marker; lane 2, purified MBP protein; lane 3, purified MUC1-MBP fusion protein; lane 4, purified MUC1 peptide. (B and C) Purified proteins were analyzed using western blot analysis with (B) anti-MBP and (C) anti-MUC1 antibodies. Lane 1, MBP protein; lane 2, MUC1-MBP fusion protein; lane 3, MUC1 peptide. MUC1, mucin 1; MBP, maltose-binding protein.

MUC1-specific CTL cytotoxicity assay. MUC1-specific CTL cytotoxicity was measured using a lactate dehydrogenase (LDH)-release assay (Promega Corporation, Madison, WI, USA). Splenic mononuclear cells were cultured in IMDM containing $100 \mathrm{U} / \mathrm{ml} \mathrm{IL-2}$ and $20 \mu \mathrm{g} / \mathrm{ml} \mathrm{MUC1} \mathrm{synthetic}$ peptide at $37^{\circ} \mathrm{C}$ in $5 \% \mathrm{CO}_{2}$ for five days. These cells were used as effectors. The B16-MUC1 or B16-neo target cells were plated at a density of $1 \times 10^{4}$ cells/well in 96 -well plates and the effector cells were added to triplicate wells according to the manufacturer's instructions. The effector-to-target cell (E/T) ratios investigated were 50:1, 25:1 and 12.5:1. Cells were incubated for $5 \mathrm{~h}$ at $37^{\circ} \mathrm{C}$ in an atmosphere of $5 \% \mathrm{CO}_{2}$. The culture supernatant $(50 \mu \mathrm{l} /$ well $)$ from each well was then transferred to a fresh 96-well plate. An LDH detection solution was added to each well (50 $\mu \mathrm{l} /$ well) and incubated in the dark for $30 \mathrm{~min}$ at room temperature, prior to the addition of the termination solution ( $50 \mu \mathrm{l} /$ well). The absorbance was measured at $490 \mathrm{~nm}$ using an ELISA reader. The percentage of CTL target-killing activity was calculated as follows: (effectors and target mixture - effectors spontaneous - target spontaneous)/(target maximum - target spontaneous) x 100 .

NK cytotoxicity assay. Splenic NK cell cytotoxicity was measured using an LDH-release assay (Promega Corporation) as described previously (28). In brief, splenic mononuclear cells from immunized mice were harvested as effectors and YAC-1 cells were used as target cells. The target cells were plated in 96-well plates at a density of $1 \times 10^{4}$ cells/well. Effector cells were added at different ratios (100:1, 50:1 and 25:1) and incubated for $5 \mathrm{~h}$ at $37^{\circ} \mathrm{C}$ in an atmosphere of $5 \% \mathrm{CO}_{2}$. The presence of $\mathrm{LDH}$ in the culture supernatant was detected as described above.

Prophylactic and therapeutic activity in C57BL/6 mice. The antitumor activity of the combination of MUC1-MBP with BCG was investigated using B16-MUC1 and B16-neo mouse melanoma models. Mice were randomly divided into seven groups of five animals, which were treated with the following agents: PBS, BCG, MBP, MUC1, MUC1/BCG, MUC1-MBP and MUC1-MBP/BCG. For the prophylactic experiments, mice were immunized three times at one-week intervals according to the aforementioned method. Five days after the final immunization, mice were subcutaneously inoculated with $2 \times 10^{6} \mathrm{~B} 16-\mathrm{MUC1}$ or B16-neo cells. For the tumor therapy experiments, mice were subcutaneously inoculated with $2 \times 10^{6}$ B16-MUC1 or B16-neo cells, prior to immunization. After four days, mice were immunized according to the aforementioned method. Tumor size was measured using calipers every three days and tumor nodule volumes were calculated according to the following formula: (longest diameter) $\mathrm{x}$ (shortest diameter) ${ }^{2} / 2$ (28).

Statistical analysis. Data are presented as the mean \pm standard deviation. One-way analysis of variance was used to compare significant differences between the means of all treatment groups. A two-sided Student's t-test was used to compare the means of individual treatments when the primary outcome was statistically significant. A value of $\mathrm{P}<0.05$ was considered to indicate a statistically significant difference. All statistical analyses were performed using SPSS 13.0 software (SPSS, Inc., Chicago, IL, USA).

\section{Results}

Expression and purification of the MUC1 peptide, MBP protein and MUC-MBP fusion protein. MBP has been utilized as a chaperone in various experimental anti-pathogenic vaccines and recombinant MBP-protein vaccines have been found to display enhanced immunogenicity $(26,27)$. In order to investigate effective cancer vaccines, a recombinant human MUC1-MBP fusion protein $(62 \mathrm{kDa})$ and the MBP protein (42 kDa) were successfully expressed in pMAL-MUC1 or pMAL-c2-transformed DH5 $\alpha$ cells using IPTG induction and purified using amylose resin affinity chromatography (Fig. 1A). The MUC1 peptide (19 kDa) was obtained following Xa Factor protease cleavage of the MUC1-MBP fusion protein (Fig. 1A). These proteins were of very high purity (>95\%) and were verified using western blot analysis with anti-MBP (Fig. 1B) or -MUC1 monoclonal antibodies (Fig. 1C).

Combined immunization with the MUC1-MBP fusion protein and BCG induces a MUC1-specific IgG2c antibody response. To determine whether the MUC1-MBP fusion 


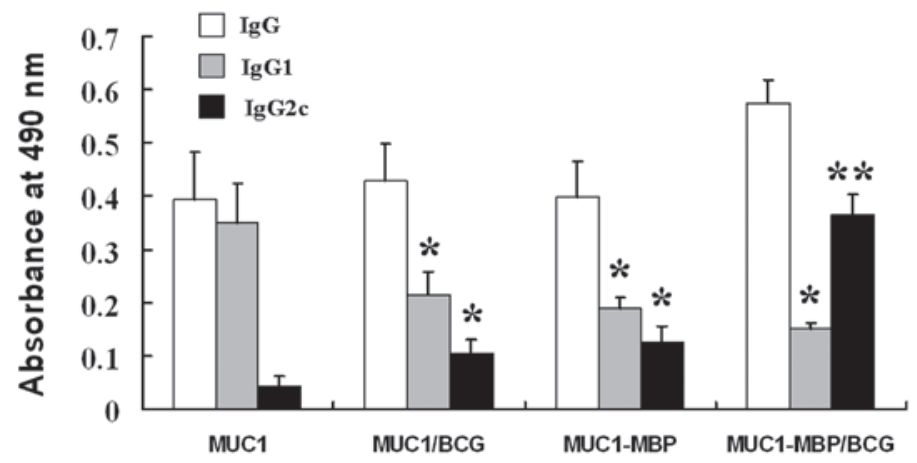

Figure 2. Analysis of MUC1-specific IgG and IgG subclasses in mouse sera. Sera from mice immunized with MUC1, MUC1/BCG, MUC1-MBP and MUC1-MBP/BCG were collected five days after the final immunization. MUC1-specific IgG, IgG1 and IgG2c in the sera at a dilution of 1:500 were assayed using ELISA. Values were determined by measuring the absorbance at $490 \mathrm{~nm}$. All values represent the mean \pm standard deviation from five mice. ${ }^{*}<0.05$ vs. $\mathrm{MUC1}$ group; ${ }^{* *} \mathrm{P}<0.01$ vs. all test groups. MUC1, mucin 1; MBP, MBP, maltose-binding protein; IgG, immunoglobulin G; BCG, Bacillus Calmette-Guerin.

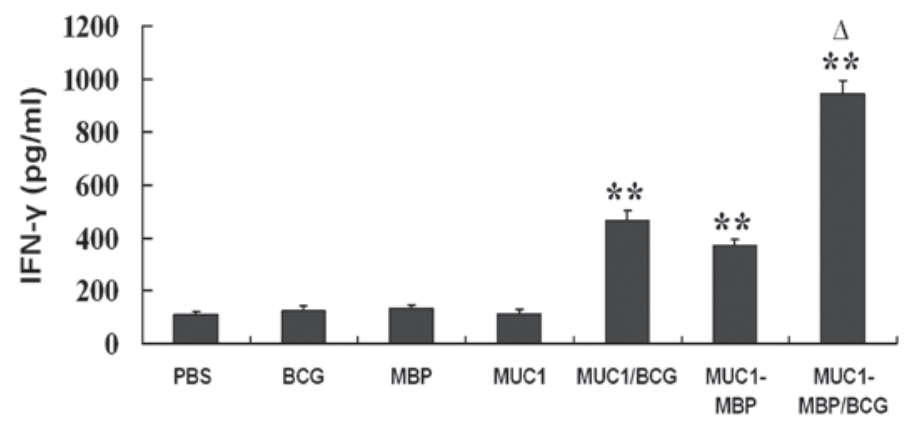

Figure 3. IFN- $\gamma$ production by Th1 cells in response to MUC1. Splenic mononuclear cells from mice immunized with PBS, BCG, MBP, MUC1, MUC1/BCG, MUC1-MBP or MUC1-MBP/BCG were cultured in Iscove's Modified Dulbecco's Media containing $100 \mathrm{U} / \mathrm{ml}$ interleukin-2 and co-cultured with or without $20 \mu \mathrm{g} / \mathrm{ml} \mathrm{MUC1}$ synthetic peptide for five days. IFN- $\gamma$-producing cells were assessed using ELISA. Cytokine levels were calculated as the cytokine levels detected upon stimulation with the MUC1 synthetic peptide minus the cytokine levels detected upon stimulation with free MUC1 synthetic peptide. Values are presented as the mean \pm standard deviation from five mice. ${ }^{* *} \mathrm{P}<0.01$ vs. $\mathrm{PBS}$ group; ${ }^{\wedge} \mathrm{P}<0.05$ vs. MUC1/BCG and MUC-MBP groups. IFN, interferon; MUC1, mucin 1; PBS, phosphate-buffered saline; BCG, Bacillus Calmette-Guerin; MBP, maltose-binding protein.

protein induces a MUC1 antibody response, the presence of anti-MUC1 IgG antibodies was examined in mouse serum using ELISA. MUC1-specific IgG antibodies were observed in mice immunized with MUC1, MUC1/BCG, MUC1-MBP and MUC1-MBP/BCG (Fig. 2).

The antibody subclass induced by the immunization reflects the relative contributions of the Th1- and Th2 immune responses. Therefore, Th2-associated MUC1-specific IgG1 and Th1-associated MUC1-specific IgG2c antibody subclasses were measured. As shown in Fig. 2, immunization with MUC1 was only observed to induce MUC1-specific IgG1 production. By contrast, immunization with MUC1/BCG and MUC1-MBP induced significantly lower levels of IgG1 and significantly higher levels of IgG2c compared with the MUC1 group $(\mathrm{P}<0.05)$. MUC1-MBP/BCG immunization induced the highest levels of $\mathrm{IgG} 2 \mathrm{c}$ and the lowest levels of IgG1 among all of the test groups. These findings indicate that MUC1 alone induces a Th2 response, whereas MUC1/BCG and MUC1-MBP induce Th1 and Th2 responses. MUC1-MBP/BCG further shifted towards a Th1 profile.

Combined immunization with the MUC1-MBP fusion protein and BCG induces Th1 cell activation. To determine whether
MUC1-MBP/BCG induces Th1 cell activation, mouse splenic mononuclear cells were isolated from immunized mice and incubated with or without the MUC1 synthetic peptide for five days. IFN- $\gamma$ secretion was then measured using ELISA. Compared with the MUC1 group, MUC1/BCG, MUC1-MBP and MUC1-MBP/BCG vaccination was found to significantly increase the production of IFN- $\gamma(\mathrm{P}<0.01)$ (Fig. 3). However, vaccination with MUC1-MBP in combination with BCG significantly increased the levels of IFN- $\gamma$ compared with vaccination with MUC1/BCG or MUC1-MBP alone ( $\mathrm{P}<0.05)$ (Fig. 3). These findings suggested that the combination of MUC1-MBP with BCG promoted MUC1-specific Th1 activation.

Combined immunization with the MUC1-MBP fusion protein and BCG induces specific CTL activity against MUCl. CTL killing activity is a gold standard measurement used to determine the efficacy of a tumor vaccine. To detect whether MUC1-MBP immunization is capable of inducing MUC1-specific CTL activity in mice, a B16 mouse melanoma cell line stably expressing human MUC1, as well as a B16-neo negative control cell line were established. Flow cytometric analysis revealed that the B16-MUC1 cells were $98.5 \%$ positive for MUC1, while the B16-neo cells were $1.5 \%$ positive for MUC1 (Fig. 4A). 
A

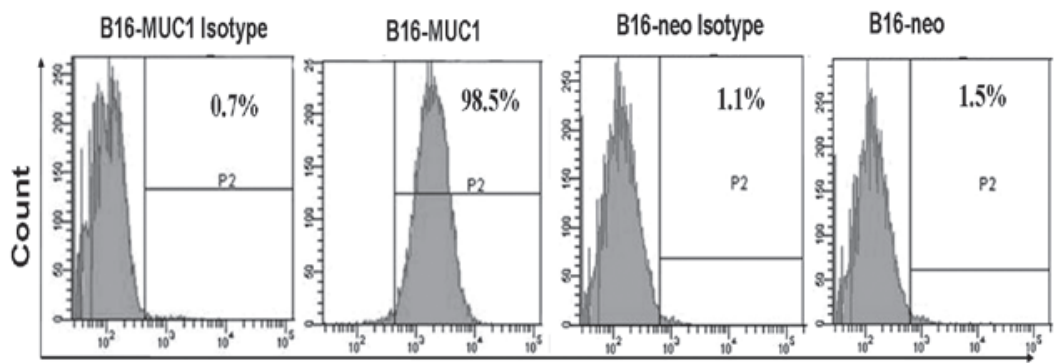

Fluorescence intensity

B

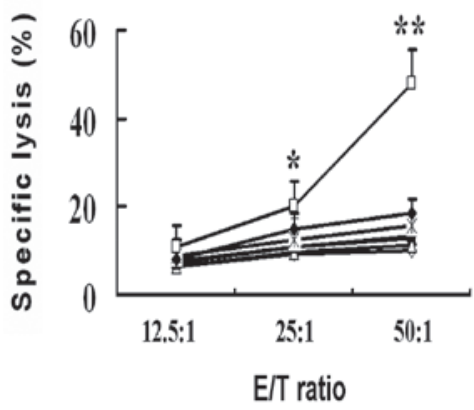

C

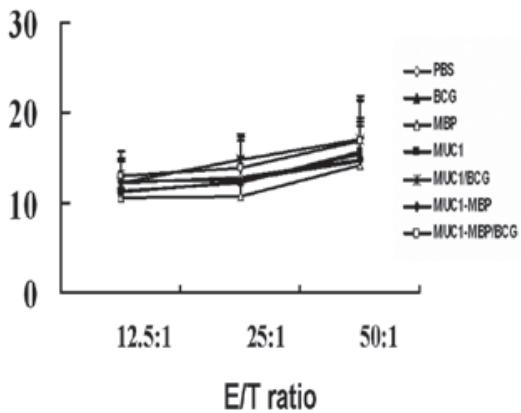

Figure 4. MUC1-MBP/BCG-induced MUC1-specific CTL cytotoxic activity. Splenic mononuclear cells from mice immunized with PBS, BCG, MBP, MUC1, MUC1/BCG, MUC1-MBP or MUC1-MBP/BCG were cultured in Iscove's Modified Dulbecco's Media containing 100 U/ml interleukin-2 and co-cultured with $20 \mu \mathrm{g} / \mathrm{ml}$ MUC1 synthetic peptide for five days and were used as effector cells. Either B16 cells transfected with the pcDNA3-MUC1 plasmid (B16-MUC1), containing full-length human MUC1 consisting of 22 variable number tandem repeats or B16 cells transfected with the pcDNA3 empty plasmid (B16-neo) were used as the target cells. (A) Flow cytometric analysis revealed that B16-MUC1 cells were 98.5\% positive for MUC1, while B16-neo cells were only 1.5\% positive for MUC1. (B and C) MUC1-specific CTL cytotoxic activity was detected at various E/T ratios using the lactate dehydrogenase release assay with either (B) B16-MUC1 target cells or (C) B16-neo target cells. Values are presented as the mean \pm standard deviation from five mice. ${ }^{*} \mathrm{P}<0.05$, ${ }^{* *} \mathrm{P}<0.01$ vs. the other groups. MUC1, mucin 1; CTL, cytotoxic T lymphocyte; MBP, maltose-binding protein; BCG, Bacillus Calmette-Guerin; E/T, effector-to-target; PBS, phosphate-buffered saline.

CTL cytotoxicity against B16-MUC1 and B16-neo cells was measured using the LDH-release assay. Lymphocytes from immunized mice were isolated in mouse lymphocyte separation medium and were used as effector cells. These effector cells were then stimulated with the MUC1 synthetic peptide for five days. B16-MUC1 or B16-neo cells were used as the target cells. The killing activity of CTL cells against the B16-MUC1 cells was observed to be significantly increased in the MUC1-MBP/BCG-immunized group compared with the other groups at $\mathrm{E} / \mathrm{T}$ ratios of 50:1 and 25:1 $(\mathrm{P}<0.01$ and $\mathrm{P}<0.05$, respectively; Fig. 4B). Cytotoxicity against B16-neo cells was not observed in any of the test groups (Fig. 4C). These findings suggest that MUC1-MBP/BCG immunization induces MUC1-specific CTL activity, while MUC1/BCG and MUC1-MBP vaccines do not.

MUC1-MBP and BCG act synergistically on NK cell cytotoxicity. NK cells are capable of killing early-stage tumor cells and we previously demonstrated that MBP is capable of inducing NK cell activation (28). Therefore, in the present study, splenic mononuclear cells were isolated from immunized mice as effector cells and NK-sensitive YAC-1 cells were used as target cells. The effector and target cells were co-incubated for $5 \mathrm{~h}$ and the culture supernatant was collected and tested for NK cell cytotoxicity using an LDH-release assay. Compared with the PBS control group, NK cell cytotoxic activity was significantly increased in the BCG, MBP, MUC1/BCG and MUC1-MBP groups at an E/T ratio of 100:1 $(\mathrm{P}<0.05)$. Furthermore, in the MUC1-MBP/BCG group, NK cell cytotoxicity was significantly higher at $\mathrm{E} / \mathrm{T}$ ratios of 100:1 and 50:1 ( $\mathrm{P}<0.01$ and $\mathrm{P}<0.05$, respectively) compared with the PBS control group (Fig. 5). These findings demonstrate that BCG, MBP and MUC1-MBP induce NK cell activation and that MUC1-MBP and BCG act synergistically on NK cells.

Combined immunization with the MUC1-MBP fusion protein and $B C G$ has an antitumor effect in vivo. To examine the antitumor activity of the MUC1-MBP fusion protein, carcinoma models were established using B16-MUC1 cells that were $98.5 \%$ positive for $\mathrm{MUC1}$ and B16-neo cells as negative controls. To determine whether the vaccines had a protective effect against tumors, female C57BL/6 mice were immunized three times at one-week intervals and were then treated with B16-MUC1 or B16-neo cells five days after the final immunization. Tumor volume was measured every three days.

B16-MUC1 tumor growth was monitored for 21 days. After nine days, the B16-MUC1 tumors in 


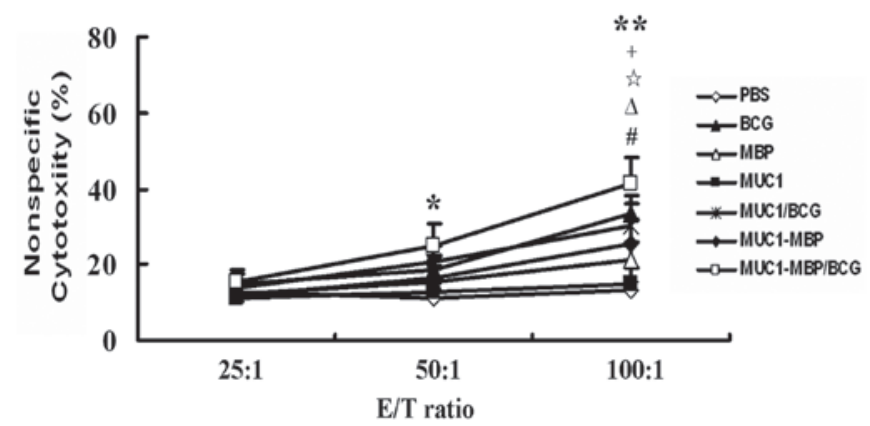

Figure 5. Combined immunization with MUC1-MBP and BCG induced synergistic NK cell cytotoxicity. Splenic mononuclear effector cells were obtained from mice immunized with PBS, BCG, MBP, MUC1, MUC1/BCG, MUC1-MBP or MUC1-MBP/BCG and co-cultured with YAC-1 cells as target cells for $5 \mathrm{~h}$. NK cell cytotoxicity was detected at various E/T ratios using the lactate dehydrogenase-release assay. Values are presented as the mean \pm standard deviation from five mice. For the ${ }^{\#} \mathrm{BCG},{ }^{\Delta} \mathrm{MBP}$, ${ }^{\sqrt{4}} \mathrm{MUC1} / \mathrm{BCG},{ }^{+} \mathrm{MUC1}-\mathrm{MBP}$ and ${ }^{*} \mathrm{MUC1}-\mathrm{MBP} / \mathrm{BCG}$ groups, $\mathrm{P}<0.05$ vs. PBS group; for the ** MUC1-MBP/BCG group, $\mathrm{P}<0.01$ vs. $\mathrm{PBS}$ group. MUC1, mucin 1; NK, natural killer; MBP, maltose-binding protein; $\mathrm{BCG}$, Bacillus Calmette-Guerin; E/T, effector-to-target; PBS, phosphate-buffered saline.

the BCG-, MBP-, MUC1/BCG-, MUC1-MBP- and MUC1-MBP/BCG-immunized mice were observed to grow less rapidly and display significantly lower sizes compared with those in the PBS-immunized mice $(\mathrm{P}<0.05)$. However, 15 days after the subcutaneous injection of the B16-MUC1 cells, the tumors were found to grow more rapidly in the mice in all of the groups except for those in the MUC1-MBP/BCG group as compared with the PBS control group (Fig. 6A). The average B16-MUC1 tumor weight was $3.87 \pm 1.01 \mathrm{~g}$ in the mice in the PBS group compared with $1.51 \pm 0.67 \mathrm{~g}$ in those in the MUC1-MBP/BCG group ( $\mathrm{P}<0.05$; Fig. 6C). As shown in Fig. 6B, B16-neo tumor growth was monitored for 18 days. After nine days, the B16-neo tumors in the mice in the BCG-, MBP-, MUC1/BCG-, MUC1-MBP- and MUC1-MBP/BCG-immunized groups displayed significantly lower volumes compared with those in the mice in the PBS-immunized group $(\mathrm{P}<0.05)$. However, by 18 days, no significant differences were observed in B16-neo tumor volume and weight between the groups (Fig. 6B and D). These findings demonstrate that the combination of MUC1-MBP and BCG significantly inhibits B16-MUC1 cell growth, while BCG, MBP, MUC1/BCG and MUC1-MBP suppress tumor growth of B16-MUC1 and B16-neo only in the early stages of tumor development.

In order to determine whether the vaccines had the potential to treat tumor growth, female C57BL/6 mice were subcutaneously inoculated with either B16-MUC1 or B16-neo cells on day 0 and then immunized with PBS, BCG, MBP, MUC1, MUC1/BCG, MUC1-MBP or MUC1-MBP/BCG on days five and 12. Tumor growth was monitored for 18 days after tumor inoculation. Similar effects to those in the tumor protection experiments were observed (Fig. 7); however, the inhibition of tumor growth was not as significant as the observed protective effects. These findings indicate that specific T-cell immunity has an important role in the antitumor activity, while specific humoral immunity has a less important role. Natural immunity may only have an effect during the early stages of tumor development.

\section{Discussion}

Studies involving MUC1-based protein/peptide vaccines have shown that the MUC1 peptide alone is incapable of inducing a cellular immune response (16). However, the cellular immune response has an important role in eliminating cancer cells. Therefore, the present study aimed to develop an efficient MUC1 protein vaccine through generating a recombinant fusion protein consisting of MUC1 (140 amino acids) and MBP. In brief, a MUC1 cDNA fragment containing seven tandem repeats was inserted into the pMAL-c2 plasmid. MUC1 peptides (140 amino acids) and MBP proteins were also generated as controls. The immune responses to MUC1-MBP, MUC1 and MBP were then assessed in mice.

In the mice immunized with MUC1, high MUC1 antibody production and a predominantly Th2-associated IgG1 response were observed. However, in the mice immunized with MUC1-MBP, higher levels of the Th1-associated IgG2c isotype and lower levels of IgG1 isotope were observed compared with MUC1 alone. Similarly, levels of the Th1 cytokine IFN- $\gamma$ were observed to increase in the MUC-MBP-treated mice but not in the MUC1-treated mice. These findings suggest that MBP may be an effective immune regulatory protein which may be useful for vaccine development, due to its capacity to alter the Th1 immune response. In addition, MBP and MUC1-MBP were found to induce $\mathrm{NK}$ cell activation, suggesting that MBP enhanced MUC1 immunogenicity by inducing specific and nonspecific immunity. MBP, a component of the maltose transport system in Escherichia coli, is commonly considered to have minimal or no bioactivity. However, previous studies by our group showed that MBP promotes lymphocyte proliferation and induces Th1 and NK cell activation $(28,29)$. In these studies, MBP enhanced immune activities and retained this function when it was conjugated to MUC1 to generate the MUC1-MBP fusion protein. Therefore, MBP is an important component of the MUC1-MBP vaccine.

BCG induces a Th1-type immune response and a previous study by our group found that MBP enhances BCG-induced Th1 cell activation (29). Thus, in the present study, BCG was used as an adjuvant to further investigate the immune activities of MUC1-MBP and the MUC1 peptide in mice. MUC1-MBP/BCG was found to induce higher levels of IgG2c and IFN- $\gamma$ as compared with the MUC1/BCG group, which is indicative of a Th1-driven response. In addition, the combination of MUC1-MBP and BCG induced a stronger activation of NK cells as compared with MBP or BCG alone. These findings indicated that treatment with $\mathrm{MUC1}-\mathrm{MBP} / \mathrm{BCG}$ induced strong, MUC1-specific Th1 cell activation, as well as non-specific immunity, suggesting that MBP and BCG had synergistic effects on specific and non-specific immunity. BCG has been found to activate numerous cells, including Th1 and NK cells $(31,32)$ and has been used as an adjuvant to treat bladder cancer, malignant melanoma and lung cancer in clinical applications $(33,34)$. In the present study, BCG was found to be a critical component of the MUC1-MBP vaccine.

Th1 cells have an important role in cellular immune responses and are associated with successful antitumor responses, particularly CTL killing activity, which is a gold standard measurement used to determine the efficacy of a tumor vaccine. In the present study, MUC1/BCG and MUC1-MBP 
A

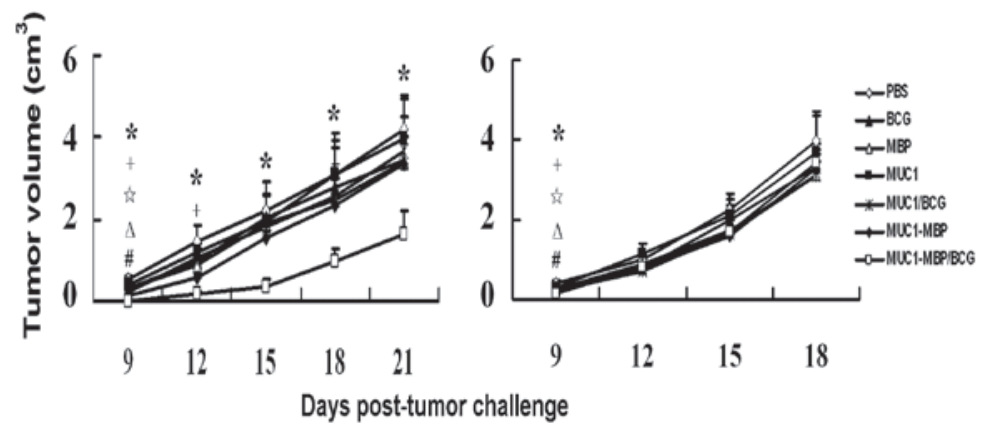

C

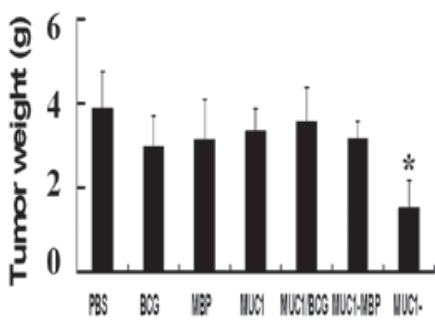

D

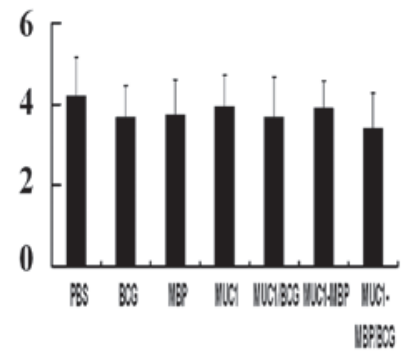

Figure 6. Prophylactic immunization of mice with the MUC1-MBP fusion protein and BCG inhibits growth in MUC1-expressing tumors. Mice were immunized with PBS, BCG, MBP, MUC1, MUC1/BCG, MUC1-MBP or MUC1-MBP/BCG three times at one-week intervals. Mice were then treated with 2x10 (A and C) B16-MUC1 or (B and D) B16-neo cells. Tumor volume was monitored at the indicated time-points and tumor weight was measured following sacrifice. Values represent the mean \pm standard deviation from five mice. For the ${ }^{\#} \mathrm{BCG},{ }^{4} \mathrm{MBP},{ }^{*} \mathrm{MUC1} / \mathrm{BCG},{ }^{+} \mathrm{MUC1}-\mathrm{MBP}$ and ${ }^{*} \mathrm{MUC1}-\mathrm{MBP} / \mathrm{BCG}$ groups, $\mathrm{P}<0.05$ vs. PBS group; for the "** MUC1-MBP/BCG group, P<0.01 vs. PBS group. MUC1, mucin 1; MBP, maltose-binding protein; BCG, Bacillus Calmette-Guerin; PBS, phosphate-buffered saline.

A

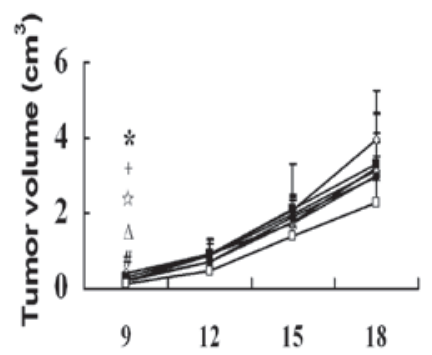

Days post-tumor challenge

B

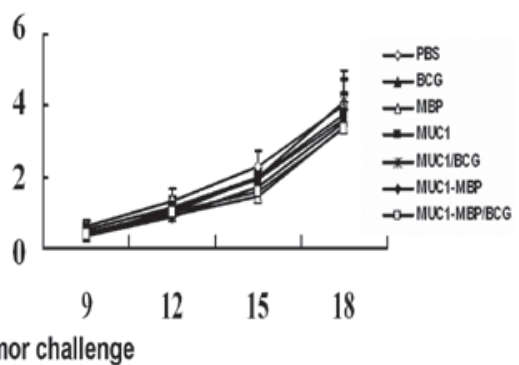

D

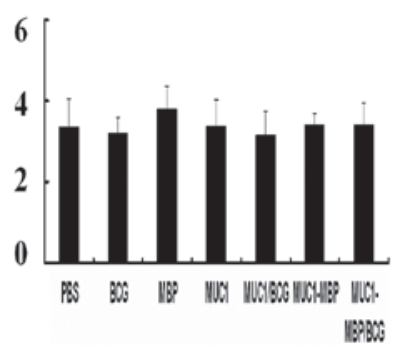

Figure 7. Therapeutic immunization of mice with the MUC1-MBP fusion protein and BCG inhibits growth in MUC1-expressing tumors. Mice were inoculated with $2 \times 10^{6}$ (A and C) B16-MUC1 or (B and D) B16-neo cells, then immunized with PBS, BCG, MBP, MUC1, MUC1/BCG, MUC1-MBP or MUC1-MBP/BCG twice. Tumor volume was monitored at the indicated time-points and tumor weight was measured following sacrifice. Each value represents the mean \pm standard deviation from five mice. For the ${ }^{\#} \mathrm{BCG},{ }^{\Delta} \mathrm{MBP},{ }^{\wedge} \mathrm{MUC1} / \mathrm{BCG},{ }^{+} \mathrm{MUC1}-\mathrm{MBP}$ and ${ }^{*} \mathrm{MUC1}-\mathrm{MBP} / \mathrm{BCG}$ groups, $\mathrm{P}<0.05$ vs. PBS group; for the ${ }^{* *} \mathrm{MUC1}$-MBP/BCG group, $\mathrm{P}<0.01$ vs. PBS group. MUC1, mucin 1; MBP, maltose-binding protein; BCG, Bacillus Calmette-Guerin; PBS, phosphate-buffered saline. 
were not sufficient to induce effective CTL activity. However, MUC1-MBP/BCG was found to induce MUC1-specific CTL activation in mice. These findings indicate that the efficacy of MUC1-MBP/BCG as a cancer vaccine involves three necessary components: (i) BCG, due to its capacity to activate Th1 cells; (ii) MBP, due to its capacity to enhance the immunogenicity of MUC1 and BCG-induced Th1 cell activation and (iii) the specific target MUC1.

The protective and therapeutic effects of MUC1-MBP/BCG on tumor growth were also investigated in mice. Immunization with BCG, MBP, MUC1/BCG, MUC1-MBP or MUC1-MBP/BCG was found to inhibit $\mathrm{MUC1}^{+} \mathrm{B} 16$ and MUC1- B16 cell growth in early mouse melanoma models, corresponding with the activation of the innate immune response in these mice. Furthermore, only MUC1-MBP/BCG immunization was observed to inhibit growth in the $\mathrm{MUCl}^{+}$ B16 cells in the late mouse melanoma model and no significant effect was observed on the MUC1 $^{-}$B16 cells. These findings suggest that the enhanced specific cellular immunity induced by MUC1-MBP/BCG is essential to inhibit tumor growth. Moreover, MUC1-MBP/BCG was found to have better prophylactic than therapeutic efficacy, suggesting that MUC1-MBP/BCG treatment may have beneficial effects for early-phase or postoperative residual tumors, but may have little effect on later phase tumors.

In conclusion, the present study showed that the combination of MUC1-MBP and BCG not only induced a specific antibody response, but also induced strong specific cellular and innate immunity. Furthermore, immunization with MUC1-MBP and BCG was observed to significantly inhibit the growth of $\mathrm{MUC1}^{+}$tumors in a mouse melanoma model. Thus, the combination of MUC1-MBP and BCG may be a promising cancer vaccine for patients with cancer.

\section{Acknowledgements}

The authors would like to thank Dr O.J. Finn (University of Pittsburgh, Pittsburgh, PA, USA) for the pcDNA3-MUC1 plasmid, which was used to transfect the B16 cells. This study was supported by grants from the Science and Technology Development Program of Jilin Province (no. 20080931), the Major Development Program for New Drugs of the Chinese Academy of Sciences during the 12th Five-Year Plan Period (no. 2011ZX09102-001-36) and the National Natural Science Foundation of China (nos. 30972782 and 81202031).

\section{References}

1. Gendler SJ, Lancaster CA, Taylor-Papadimitriou J, et al: Molecular cloning and expression of human tumor-associated polymorphic epithelial mucin. J Biol Chem 265: 15286-15293, 1990.

2. Gendler S, Taylor-Papadimitriou J, Duhig T, et al: A highly immunogenic region of a human polymorphic epithelial mucin expressed by carcinomas is made up of tandem repeats. J Biol Chem 263: 12820-12823, 1998.

3. Apostolopoulos V, Pietersz GA and McKenzie IF: MUC1 and breast cancer. Curr Opin Mol Ther 1: 98-103, 1999.

4. Woenckhaus M, Merk J, Stoehr R, et al: Prognostic value of FHIT, CTNNB1, and MUC1 expression in non-small cell lung cancer. Hum Pathol 39: 126-136, 2008.

5. Cozzi PJ, Wang J, Delprado W, et al: MUC1, MUC2, MUC4, MUC5AC and MUC6 expression in the progression of prostate cancer. Clin Exp Metastasis 22: 565-573, 2005.
6. Kigure S: Immunohistochemical study of the association between the progression of pancreatic ductal lesions and the expression of MUC1, MUC2, MUC5AC, and E-cadherin. Rinsho Byori 54: 447-452, 2006.

7. Brossart P, Schneider A, Dill P, Schammann T, et al: The epithelial tumor antigen MUC1 is expressed in hematological malignancies and is recognized by MUC1-specific cytotoxic T-lymphocytes. Cancer Res 61: 6846-6850, 2001.

8. Kawano T, Ito M, Raina D, et al: $\mathrm{MUC1}$ oncoprotein regulates Bcr-Abl stability and pathogenesis in chronic myelogenous leukemia cells. Cancer Res 67: 11576-11584, 2007.

9. Hollingsworth MA and Swanson BJ: Mucins in cancer: protection and control of the cell surface. Nat Rev Cancer 4: 45-60, 2004

10. Kobayashi M, Iwamatsu A, Shinohara-Kanda A, et al: Activation of ErbB3-PI3-kinase pathway is correlated with malignant phenotypes of adenocarcinomas. Oncogene 22: 1294-1301, 2003.

11. Pisarev VM, Kinarsky L, Caffrey T, et al: T cells recognize $\mathrm{PD}(\mathrm{N} / \mathrm{T}) \mathrm{R}$ motif common in a variable number of tandem repeat and degenerate repeat sequences of MUC1. Int Immunopharmacol 5: 315-330, 2005.

12. Yang E, Hu XF and Xing PX: Advances of MUC1 as a target for breast cancer immunotherapy. Histol Histopathol 22: 905-922, 2007.

13. Tarp MA and Clausen H: Mucin-type O-glycosylation and its potential use in drug and vaccine development. Biochim Biophys Acta 1780: 546-563, 2008.

14. Mukherjee P, Pathangey LB, Bradley JB, et al: MUC1-specific immune therapy generates a strong anti-tumor response in a MUC1-tolerant colon cancer model. Vaccine 25: 1607-1618, 2007.

15. Tang CK, Sheng CK, Pouniotis D, et al: Oxidized and reduced mannan mediated MUC1 DNA immunization induce effective anti-tumor responses. Vaccine 26: 3827-3834, 2008.

16. Pejawar-Gaddy S, Rajawat Y, Hilioti Z, et al: Generation of a tumor vaccine candidate based on conjugation of a MUC1 peptide to polyionic papillomavirus virus-like particles. Cancer Immunol Immunother 59: 1685-1696, 2010.

17. Deguchi T, Tanemura M, Miyoshi E, et al: Increased immunogenicity of tumor-associated antigen, mucin 1, engineered to express alpha-gal epitopes: a novel approach to immunotherapy in pancreatic cancer. Cancer Res 70: 5259-5269, 2010.

18. Choi DH, Woo JK, Choi Y, et al: A novel chimeric DNA vaccine: Enhancement of preventive and therapeutic efficacy of DNA vaccine by fusion of Mucin 1 to a heat shock protein 70 gene. Mol Med Rep 4: 885-890, 2011.

19. Yang H, Cho NH and Seong SY: The Tat-conjugated N-terminal region of mucin antigen 1 (MUC1) induces protective immunity against MUC1-expressing tumours. Clin Exp Immunol 158: 174-185, 2009.

20. Sangha R and Butts C: L-BLP25: a peptide vaccine strategy in non small cell lung cancer. Clin Cancer Res 13: s4652-s4654, 2007.

21. Apostolopoulos V, Pietersz GA, Tsibanis A, et al: Pilot phase III immunotherapy study in early-stage breast cancer patients using oxidized mannan-MUC1 [ISRCTN71711835]. Breast Cancer Res 8: R27, 2006.

22. Holmberg LA and Sandmaier BM: Vaccination with Theratope (STn-KLH) as treatment for breast cancer. Expert Rev Vaccines 3: 655-663, 2004

23. Tang Y, Zhang L, Yuan J, et al: Multistep process through which adenoviral vector vaccine overcomes anergy to tumor-associated antigens. Blood 104: 2704-2713, 2004.

24. Boos W and Shuman H: Maltose/maltodextrin system of Escherichia coli: transport, metabolism, and regulation. Microbiol Mol Biol Rev 62: 204-229, 1998.

25. Riggs P: Expression and purification of recombinant proteins by fusion to maltose-binding protein. Mol Biotechnol 15: 51-63, 2000.

26. Kang QZ, Duan GC, Fan QT, et al: Fusion expression of Helicobacter pylori neutrophil-activating protein in E. coli, World J Gastroenterol 11: 454-456, 2005.

27. Fernandez S, Palmer DR, Simmons M, et al: Potential role for Toll-like receptor 4 in mediating Escherichia coli maltose-binding protein activation of dendritic cells. Infect Immun 75: 1359-1363, 2007.

28. Zhang Q, Ni W, Zhao X, et al: Synergistic antitumor effects of Escherichia coli maltose binding protein and Bacillus Calmette-Guerin in a mouse lung carcinoma model. Immunol Lett 136: 108-113, 2011. 
29. Zhao XX, Ma JC, Fang F, et al: Effect of Escherichia coli maltose-binding protein on mouse Th1 cell activation. Chin J Immunol 25: 504-507, 2009.

30. Pereira HM, Cleasby A, Pena S SD, et al: Cloning, expression and preliminary crystallographic studies of the potential drug target purine nucleoside phosphorylase from Schistosoma mansoni. Acta Crystallogr D Biol Crystallogr 59: 1096-1099, 2003.

31. Vetskova EK, Muhtarova MN, Avramov TI, et al: Immunomodulatory effects of BCG in patients with recurrent respiratory papillomatosis. Folia Med (Plovdiv) 55: 49-54, 2013.
32. Cautivo KM,Bueno SM,Cortes CM, et al: Efficient lung recruitment of respiratory syncytial virus-specific Th1 cells induced by recombinant bacillus Calmette-Guerin promotes virus clearance and protects from infection. J Immunol 185: 7633-7645, 2010.

33. Kawai K, Miyazaki J, Joraku A, et al: Bacillus Calmette-Guerin (BCG) immunotherapy for bladder cancer: current understanding and perspectives on engineered BCG vaccine. Cancer Sci 104: 22-27, 2013

34. Sánchez Olivas MA, Valencia Zavala MP, Montes Montes J, et al: Immunology and BCG therapeutics. Rev Alerg Mex 55: 153-163, 2008 (In Spanish). 